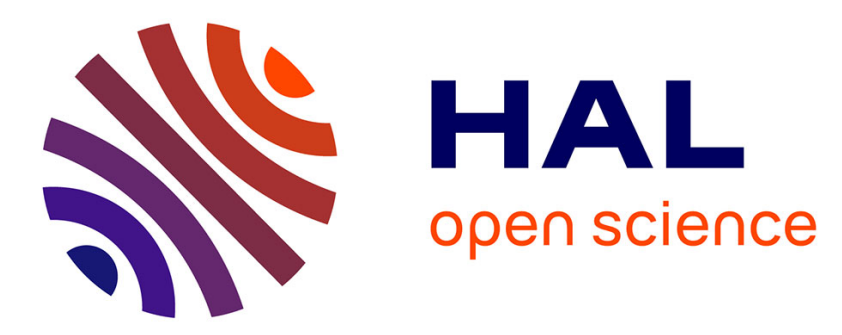

\title{
Doping of Monolayer Transition-Metal Dichalcogenides via Physisorption of Aromatic Solvent Molecules
}

Ye Wang, Amine Slassi, Marc-Antoine Stoeckel, Simone Bertolazzi, Jerôme

Cornil, David Beljonne, Paolo Samorì

\section{- To cite this version:}

Ye Wang, Amine Slassi, Marc-Antoine Stoeckel, Simone Bertolazzi, Jerôme Cornil, et al.. Doping of Monolayer Transition-Metal Dichalcogenides via Physisorption of Aromatic Solvent Molecules. Journal of Physical Chemistry Letters, 2019, 10 (3), pp.540-547. 10.1021/acs.jpclett.8b03697 . hal02056928

\section{HAL Id: hal-02056928 \\ https://hal.science/hal-02056928}

Submitted on 4 Mar 2019

HAL is a multi-disciplinary open access archive for the deposit and dissemination of scientific research documents, whether they are published or not. The documents may come from teaching and research institutions in France or abroad, or from public or private research centers.
L'archive ouverte pluridisciplinaire HAL, est destinée au dépôt et à la diffusion de documents scientifiques de niveau recherche, publiés ou non, émanant des établissements d'enseignement et de recherche français ou étrangers, des laboratoires publics ou privés. 


\section{Doping of Monolayer Transition Metal}

\section{Dichalcogenides via Physisorption of}

\section{Aromatic Solvent Molecules}

Ye Wang,' Amine Slassi, ${ }^{2}$ Marc-Antoine Stoeckel,', Simone Bertolazzi,' Jerôme Cornil, ${ }^{2}$ David Beljonne, ${ }^{*}$ Paolo Samorì *

University of Strasbourg, CNRS, ISIS UMR 7006, 8 allée Gaspard Monge, F-67000, Strasbourg, France.

Laboratory for Chemistry of Novel Materials, Université de Mons, Place du Parc 20, 7000 Mons, Belgium

\section{Corresponding Author}

*Paolo Samorì samori@unistra.fr ; David Beljonne david.beljonne@umons.ac.be

ABSTRACT Two-dimensional (2D) transition metal dichalcogenides (TMDs) recently emerged as novel materials displaying a wide variety of physico-chemical properties that render them unique scaffolds for high-performance (opto)electronics. The controlled physisorption of molecules on the TMD surface is a viable approach to tune their optical and electronic properties. Solvents, made of small aromatic molecules, are frequently employed for the cleaning of the 2D materials or as "dispersant" for their chemical functionalization with larger (macro)molecules, without considering their potential key effect in locally modifying the characteristics of $2 \mathrm{D}$ materials. In this work, we demonstrate how the electronic and optical properties of mechanically exfoliated monolayer of $\mathrm{MoS}_{2}$ and $\mathrm{WSe}_{2}$ are modified when physically interacting with small aromatic molecules of common solvents. Low- 
temperature photoluminescence (PL) spectra recorded at $78 \mathrm{~K}$ revealed that physisorbed benzene derivatives could modulate the charge carrier density in monolayer TMDs, hence affecting the switching between neutral exciton and trion (charged exciton). By combining experimental evidences with DFT calculations, we confirm that charge transfer doping on TMDs depends not only on difference in chemical potential between molecules and $2 \mathrm{D}$ materials, but also on the thermodynamic stability of physisorption. Our results provide unambiguous evidence of the great potential of optical and electrical tuning of monolayer $\mathrm{MoS}_{2}$ and $\mathrm{WSe}_{2}$ by physisorption of small aromatic solvent molecules, which is highly relevant both for fundamental studies and more device applied purposes.

Keywords: transition metal dichalcogenides, aromatic molecules, doping, photoluminescence, Density Functional Theory.

\section{TOC GRAPHICS}

KEYWORDS transition metal dichalcogenides, aromatic molecules, doping, photoluminescence, Density Functional Theory.

\section{TEXT}

During the last decade, two-dimensional (2D) materials have attracted remarkable attention owing to their unique physical and chemical properties. Among them, transition metal dichalcogenides (TMDs) are semiconducting systems that exhibit an indirect-to-direct bandgap transition from bulk to monolayer, large exciton binding energies, and inversion symmetry breaking, which are attractive for a large range of applications from (opto)electronics to valleytronics ${ }^{1-6}$. The largest surface-to-volume 
ratio in the crystal structure of monolayer TMDs makes them extremely sensitive to changes in the environment.? In this regard, the simple physisorption of atoms and molecules represents a powerful method to modulate their optical and electrical properties..$^{7.8}$

Highly polar physisorbed molecules including TCNQ and NADH, hydrazine, ${ }^{10}$ benzene viologen," have been utilized for the chemical doping of monolayer TMDs. Interactions between these molecules and TMDs induce charge transfer, thus modifying the Fermi level and tuning the electronic and optical properties of the material. In monolayer TMDs, strong electron-hole Coulomb interactions enable the formation of stable optically generated excitons at room temperature; ${ }^{12 \cdot 13}$ these excitons are building blocks for the generation of many-body bound states such as electronbound exciton (negative trion) or hole-bound exciton (positive trion) ${ }^{14-15}$. Accordingly, electron/hole concentrations modified by physisorbed molecules effectively influence the formation of excitons and trions in TMDs.<smiles>c1ccccc1</smiles><smiles>Cc1ccc(C)cc1</smiles><smiles>FC(F)(F)c1ccc(C(F)(F)F)c(C(F)(F)F)c1</smiles>

Scheme 1. Chemical formula of benzene, m-xylene, p-xylene, 1,3bis(trifluoro)methylbenzene (1,3-TFMB), and 1,4-bis(trifluoromethyl)benzene $(1,4-$ TFMB).

Although a great effort has been devoted to the non-covalent functionalization of monolayer TMDs via molecular physisorption to improve fundamental properties ${ }^{16}$ such as photoluminescence tuning, ${ }^{910,17-18}$ electron/hole doping ${ }^{1020}$ and device contact improvement, ${ }^{21}$ the simple interaction of a functionalized benzene ring (being 
frequently a fragment of solvent molecules) and monolayer TMDs has not yet been fully unraveled.2 Despite the fact that typical solvent molecules are seemingly considered as inert media, aromatic molecules have been proved to cause $\mathrm{n}$ - or $\mathrm{p}$ doping with distinct functional groups through $\pi-\pi$ interactions with graphene. ${ }^{23}$ Unfortunately, similar studies on TMDs have not yet been reported. To gain a comprehensive understanding over the effect of physisorbed molecules on monolayer TMDs, we focused here our attention to the benzene and its derivatives depicted in Scheme 1, i.e. benzene, m-xylene, p-xylene, 1,3-bis(trifluoro)methylbenzene (1,3TFMB) and 1,4-bis(trifluoromethyl)benzene (1,4-TFMB)) as aromatic molecules, and $\mathrm{MoS}_{2}$ and $\mathrm{WSe}_{2}$ as representative TMD monolayers acting as platforms for physisorption. The functionality of aromatic molecules is therefore modified through simple substitution in the meta and para position with either more apolar methyl ($\left.\mathrm{CH}_{3}\right)$ or more polar trifluoromethyl $\left(-\mathrm{CF}_{3}\right)$ groups. The melting point, boiling point and total dipole moment of each molecule are listed in in Table S1. Importantly, benzene, m-xylene and p-xylene are commonly used as solvents to dissolve complex organic molecules for functionalization of $2 \mathrm{D}$ materials and for device fabrication. These aromatic solvents could be unintentionally physisorbed on 2D materials resulting in changes in physical and chemical properties. Thus, understanding the effect of these molecules on monolayer TMDs is a crucial issue to be addressed not only for unveiling molecule-TMD interactions but also for device optimization. We reveal here on the occurrence of charge transfer between physisorbed molecules and monolayer TMDs, leads to change in electron/hole density and trion/exciton intensity ratio. Through low-temperature photoluminescence (PL) measurements performed at 78 K corroborated with Density Functional Theory calculations, we quantify n or $\mathrm{p}$ nature of doping for each molecule deposited on monolayer TMDs. Our results 
provide a text-book proof-of-concept on the use of physisorbed aromatic molecules on single-layer TMDs to dope the 2D material in a controlled fashion.

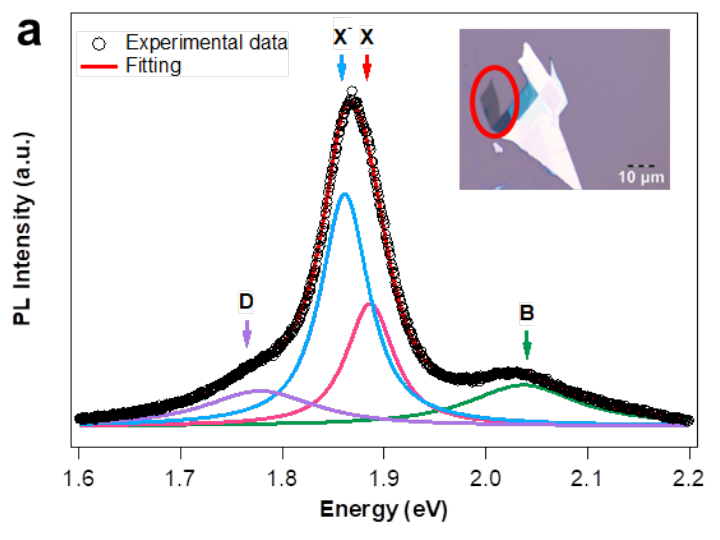

b
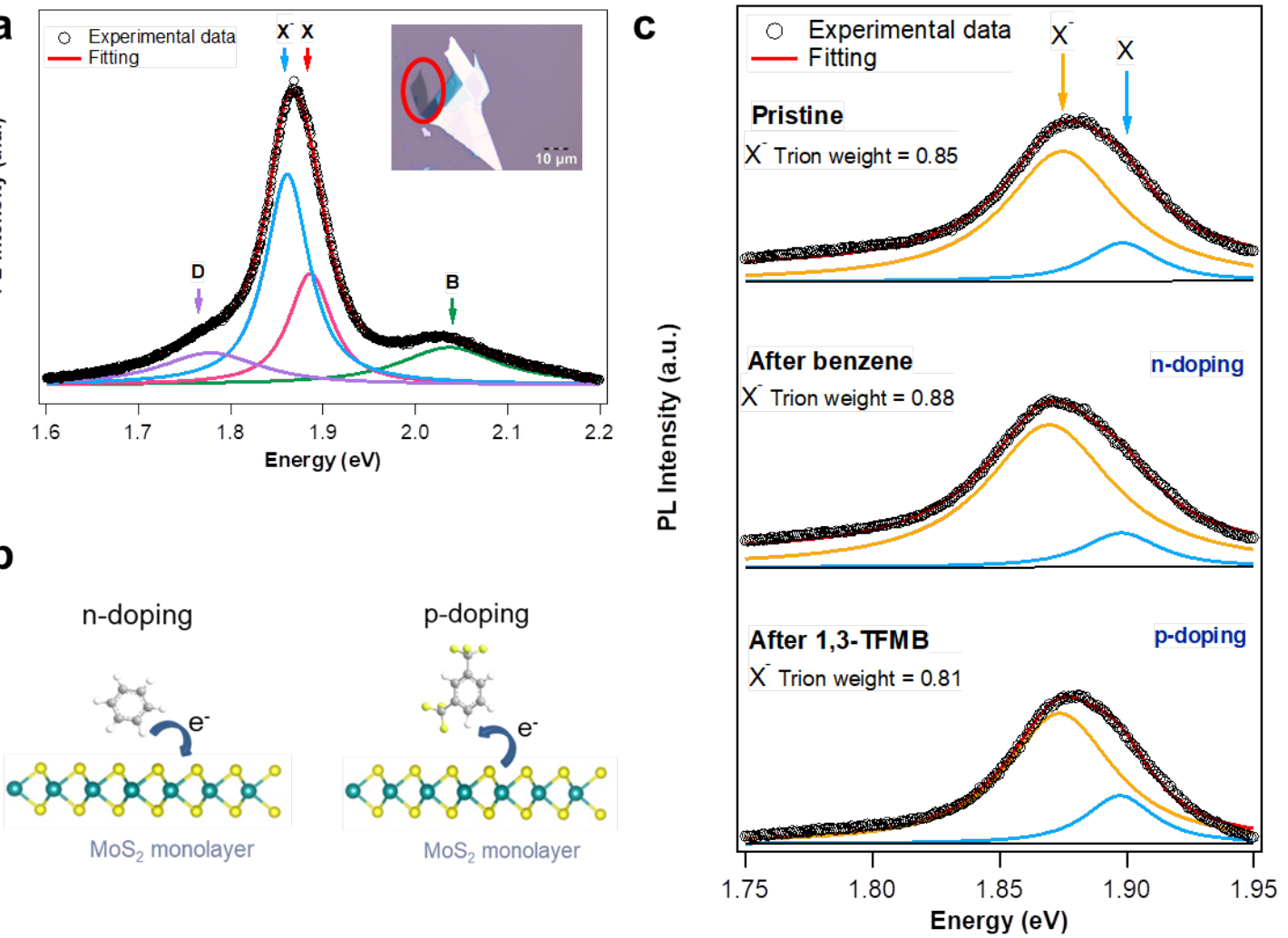

Figure 1. a) Photoluminescence spectra of monolayer $\mathrm{MoS}_{2}$ at $78 \mathrm{~K}$ (inset: optical image of as exfoliated monolayer $\mathrm{MoS}_{2}$ highlighted with a red circle. b) Molecular representation of charge transfer between physisorbed aromatic molecules and monolayer $\mathrm{MoS}_{2}$. c) Photoluminescence spectra of monolayer $\mathrm{MoS}_{2}$ before and after physisorption of benzene and 1,3-TFMB with calculated X-trion weight.

The photoluminescence behavior of monolayer $\mathrm{MoS}_{2}$ is known to be strongly temperature dependent. ${ }^{2+26}$ Typical PL spectrum of monolayer $\mathrm{MoS}_{2}$ at $78 \mathrm{~K}$ recorded in $\mathrm{N}_{2}$ atmosphere is shown in Figure 1 (a). It exhibits four components in the range of 1.6 to $2.2 \mathrm{eV}$ : a neutral A exciton $(\mathrm{X})$ at $\sim 1.88 \mathrm{eV}$ and a negatively charged $\mathrm{A}$ trion $(\mathrm{X})$ at $\sim 1.90 \mathrm{eV}$, a B exciton (B) at $\sim 2.05 \mathrm{eV}$ and a defect-induced emission (D) at $\sim 1.75 \mathrm{eV}$. The A excitons ( $\mathrm{X}$ and $\mathrm{X}$ ) are blue-shifted of $23 \mathrm{meV}$ compared to room temperature due to the bandgap enlargement upon decreasing the temperature. ${ }^{24}$ Temperature dependent studies on the photoluminescence of monolayer $\mathrm{MoS}_{2}$ (Figure S1 in the 
Supporting information) reveals that localized emission caused by excitons bounded with defects (D) appears below $100 \mathrm{~K}$. It is fair to indicate that such D peak is not observed in every flakes of our experiment likely because of the difference in defect density generated by mechanical exfoliation.

The physisorption of benzene and its derivatives (i.e. benzene, m-xylene, $\mathrm{p}$-xylene, 1,3-bis(trifluoro)methylbenzene (1,3-TFMB) and 1,4-bis(trifluoromethyl)benzene $(1,4-\mathrm{TFMB}))$ as aromatic molecules onto monolayer $\mathrm{MoS}_{2}$ (Figure 1 (b)) is explored by monitoring the photoluminescence spectra. Such study provides evidence for the occurrence of chemical doping as revealed by major changes in PL emission intensity and shape. ${ }^{9}$ Representative PL spectra of monolayer $\mathrm{MoS}_{2}$ tuned by aromatic molecules at $78 \mathrm{~K}$ are portrayed in Figure 1. a) Photoluminescence spectra of monolayer $\mathrm{MoS}_{2}$ at $78 \mathrm{~K}$ (inset: optical image of as exfoliated monolayer $\mathrm{MoS}_{2}$ highlighted with a red circle. b) Molecular representation of charge transfer between physisorbed aromatic molecules and monolayer $\mathrm{MoS}_{2}$. c) Photoluminescence spectra of monolayer $\mathrm{MoS}_{2}$ before and after physisorption of benzene and 1,3-TFMB with calculated X-trion weight. (c) whereas the spectra, and related fitting, for $\mathrm{MoS}_{2}$ with all kinds of physisorbed molecules is displayed in Figure S3 in the Supporting Information. We focus our attention on the A peak that tracks the population of trion (X) and neutral exciton (X). Given that at $78 \mathrm{~K}$ all aromatic molecules investigated are in their solid phase, the chemical doping process might differ from those reported in previous studies carried out at room temperature. ${ }^{9 \cdot 10,17-18}$ Our data reveal that upon treatment with benzene, the spectral weight of trion increases whereas the use of 1,3-TFMB induces a decrease in the trion weight. Such effects are caused by changes in charge carrier density in monolayer $\mathrm{MoS}_{2}$ induced by molecular doping. When monolayer $\mathrm{MoS}_{2}$ is $\mathrm{n}$-doped, the increase in electron density promotes the formation of negatively charged trion. 
Conversely, p-doping enables the recombination of neutral excitons into positively charged trion. The trion weight can be quantified as:

$$
\gamma^{-}=\frac{I_{X^{-}}}{I_{\text {total }}}=\frac{I_{X^{-}}}{I_{X^{-}}+I_{X}}
$$

where $\gamma^{-}$is the negative trion weight of monolayer $\mathrm{MoS}_{2}, I_{X^{-}}$is the area of negative trion peak, $I_{X}$ is the area of neutral exciton peak and $I_{\text {total }}$ is the area of total photoluminescence intensity. Therefore, we calculate the trion weight change $(\Delta \gamma)$. $\Delta \gamma^{-}>0$, indicates an increase in electron density upon molecular physisorption, implying a n-doping effect. Conversely $\Delta \gamma^{-}<0$, denotes a decrease in electron density upon molecular physisorption, corresponding to p-doping.

To explore the origin of the trion weight change $\left(\Delta \gamma^{-}\right)$, we have discussed different mechanisms which led to the exclusion of dipolar effects and the activation of defectinduced photoluminescence, as shown in Supporting notes in Supporting Information. This discussion made it possible to demonstrate that the observed induced doping in the TMDs is due to charge transfer between molecules and 2D materials. The trion weight change $\left(\Delta \gamma^{-}\right)$quantifies the ability of doping, in this specific case of $\mathrm{MoS}_{2}$ as a result of the physisorption of the molecular monolayer, as determined by PL measurements. In the simplest approximation, when two species $A$ and $B$ (here the solvent molecules and the $\mathrm{MoS}_{2}$ solid) with chemical potentials $\mu_{A} \neq \mu_{B}$ are brought into close contact, electron density $N$ flows from the high-potential to the low-potential system until equilibration is reached: ${ }^{2}$

$$
\Delta N=\frac{\mu_{A}-\mu_{B}}{\eta_{A}+\eta_{B}}
$$

where $\eta_{A}$ and $\eta_{B}$ are the chemical hardness of $\mathrm{A}$ and $\mathrm{B}$, respectively. To understand the difference in values of $\Delta \gamma^{-}$resulting from the physisorption of different 
molecules, we have calculated the chemical potential and hardness values of monolayer $\mathrm{MoS}_{2}$ and the investigated aromatic molecules. ${ }^{28}$ In the frontier molecular orbital framework and using a finite difference approximation, these write:

$$
\mu=\frac{1}{2}\left(\varepsilon_{\text {hole }}+\varepsilon_{\text {electron }}\right) ; \eta=\frac{1}{2}\left(\varepsilon_{\text {electron }}-\varepsilon_{\text {hole }}\right)
$$

Where $\varepsilon_{\text {hole }}\left(\varepsilon_{\text {electron }}\right)$ denotes the energy of the molecular HOMO (LUMO) level for the solvent and the energy of the top of the valence (conduction) band for the $2 \mathrm{D}$ solid.

As shown in Figure 2, fluorinated molecules have a smaller chemical potential due to the electron-withdrawing character of $-\mathrm{CF}_{3}$ groups. The chemical potential of monolayer $\mathrm{MoS}_{2}$ is calculated to be $-5.1 \mathrm{eV}$, which is closely above that of -5.15 and $5.19 \mathrm{eV}$ for 1,3-TFMB and 1,4-TFMB, respectively. Therefore, electrons tend to transfer from $\mathrm{MoS}_{2}$ to 1,3-TFMB and 1,4-TFMB, so that electron density is decreased in monolayer $\mathrm{MoS}_{2}$ resulting in p-doping effect. In contrast, benzene $(-3.84 \mathrm{eV}), \mathrm{m}-$ xylene $(-3.50 \mathrm{eV})$ and p-xylene $(-3.47 \mathrm{eV})$ have higher chemical potentials than monolayer $\mathrm{MoS}_{2}$, hence it is easier to transfer electron from the former to the latter, leading to $\mathrm{n}$-doping of the 2D layer. While the chemical hardness weighted difference in chemical potential between aromatic molecules explains the type of doping of $\mathrm{MoS}_{2}$, the quantitative agreement is limited. In other words, the sign of the calculated $\Delta N$ in Eq. (2) is consistent with the measured $\Delta \gamma^{-}$, but the trend with the nature of the solvent is only qualitative (see Table S2 in Supporting Information). For example, benzene has smaller chemical potential than xylenes but it gives larger trion weight change; 1,3-TFMB and 1,4-TFMB have much smaller chemical potential difference than other molecules but $\Delta \gamma^{-}$are comparable to benzene and xylenes. This is likely because of the thermodynamic stability drive to certain configurations of the self- 
assembled molecules on the surface of $\mathrm{MoS}_{2}$, possibly sourcing a surface electrostatic potential, together with other effects not included in the simple model (hybridization with and electronic polarization in the solid). To better appraise these effects, we have assessed the charge transfer taking place from the solvents to the $\mathrm{MoS}_{2}\left(\mathrm{WSe}_{2}\right)$ sheet upon adsorption by applying the Bader charge analysis to the equilibrated interfaces ${ }^{29}$. The calculated charge transfer matches the trend of $\Delta \gamma^{-}$much better than the chemical potential based model (Figure 2).

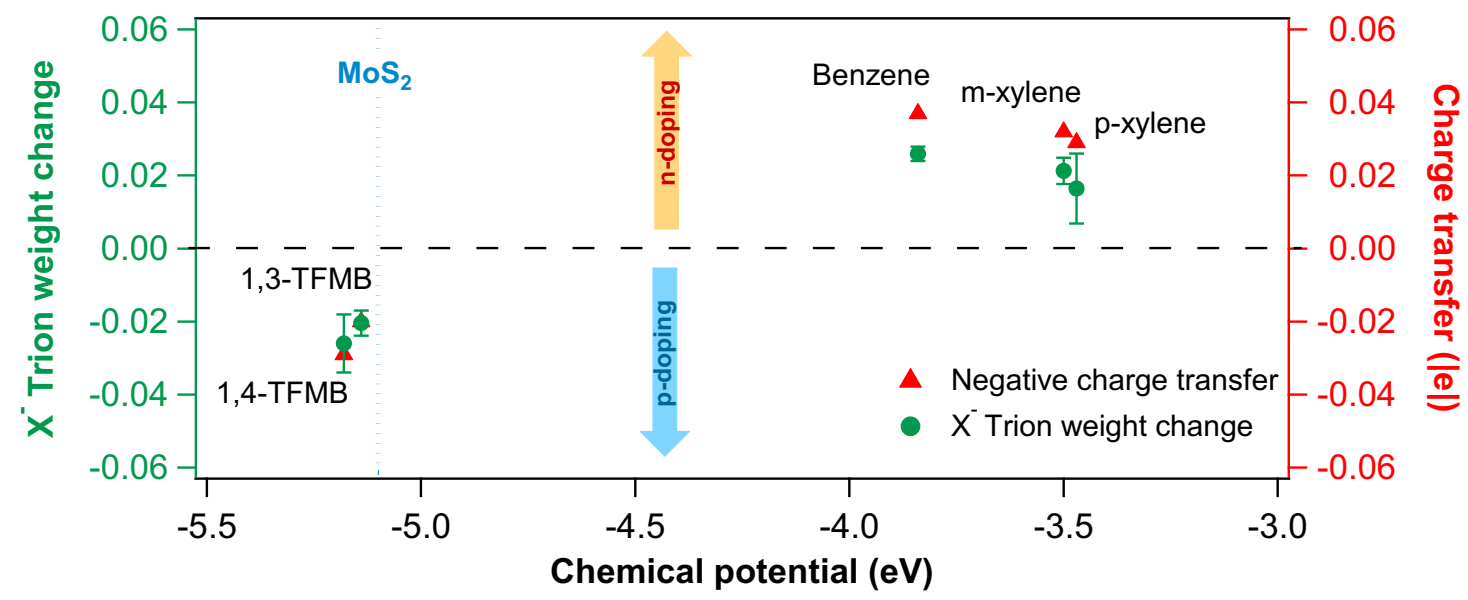

Figure 2. $\mathrm{X}^{-}$trion weight change and calculated Bader charge transfer (red) by physisorption of aromatic molecules from low-temperature PL measurement of $\mathrm{MoS}_{2}$ (green) as a function of chemical potential. Fluorinated molecules (1,3-TFMB and 1,4TFMB) possessing lower chemical potential are easier to accept electrons from monolayer $\mathrm{MoS}_{2}$; in contrast, non-fluorinated molecules (benzene, p-xylene and $\mathrm{m}$ xylene) with higher chemical potential donate electrons to monolayer $\mathrm{MoS}_{2}$.

The correlation of $\Delta \gamma^{-}$and charge transfer value can be estimated by mass action model ":

$$
\frac{N_{X} n_{e l}}{N_{X^{-}}}=\left(\frac{4 m_{X} m_{e}}{\pi \hbar^{2} m_{X^{-}}}\right) k_{B} T \exp \left(-\frac{E_{b}}{k_{B} T}\right)
$$

where $N_{X}$ and $N_{X^{-}}$are the population of excitons (X) and trions (X). $n_{e l}$ is the electron density and $E_{b}$ is the binding energy of trion $(\sim 20 \mathrm{meV})$. $\mathrm{T}$ is the temperature 
(78 K). $m_{X}, m_{X^{-}}$and $m_{e}$ are effective masses of exciton, trion and electron respectively. Considering $m_{e} \approx 0.35 m_{0}$ and $m_{h} \approx 0.45 m_{0}, m_{X}$ and $m_{X^{-}}$can be calculated as $m_{X}=m_{e}+m_{h}=0.8 m_{0}$, and $m_{X^{-}}=2 m_{e}+m_{h}=1.15 m_{0}$. Hence, the trion weight can be expressed as

$$
\frac{I_{X^{-}}}{I_{\text {total }}}=\frac{\frac{\gamma_{t r} N_{X^{-}}}{\gamma_{e x} N_{X}}}{1+\frac{\gamma_{\text {tr }} N_{X^{-}}}{\gamma_{\text {ex } N_{X}}}} \approx \frac{1.5 * 10^{-15} n_{e l}}{1+1.5 * 10^{-15} n_{e l}}
$$

where $\gamma_{t r}$ and $\gamma_{e x}$ are radiative decay rates of trion and exciton, respectively. Figure 3 demonstrates a good correspondence between the electron density change obtained from experimental data and the charge transfer predicted by DFT calculations, as the electron density change of monolayer $\mathrm{MoS}_{2}$ after the physisorption of aromatic molecules is linearly proportional to charge transfer value per molecule. By carefully excluding the doping effect from air atmosphere, our measurements shows that the modulation of charge density by solvent trace in monolayer $\mathrm{MoS}_{2}$ is largely decreased to $10^{11} / \mathrm{cm}^{-2}$ compared to previous studies that attain $10^{13} / \mathrm{cm}^{2} .^{30}$ Therefore, it is possible to estimate the number of physisorbed molecules on the surface of monolayer $\mathrm{MoS}_{2}$ by considering

$$
\Delta n_{e l} \cdot|e|=n_{m o l} \cdot \Delta \sigma^{-}
$$

where $\Delta n_{e l}$ is the change in electron density, $|e|$ is the absolute value of elementary charge, $n_{m o l}$ is the number of molecules adsorbed per unit area $\left(\mathrm{cm}^{2}\right)$ and $\Delta \sigma^{-}$is the negative charge transfer value. The estimated number of adsorbed molecules for each chemical is listed in

Table 1. It is clear that xylenes have the smaller number of adsorbed molecules, whereas fluorinated molecules tend to self-assemble more densely on the surface of the $\mathrm{MoS}_{2}$. This might be attributed to the preference of Coulomb interaction between electron-withdrawing $-\mathrm{CF}_{3}$ group and the intrinsically n-doped monolayer $\mathrm{MoS}_{2} \mathrm{We}$ 
have also studied at a theoretical level how charge transfer from the physisorbed molecules to the TMD monolayer is influenced by varying the density of molecules, inter-layer distance and inclination angle between molecules and monolayer $\mathrm{MoS}_{2}$, as discussed in the Supporting notes in the Supporting information.

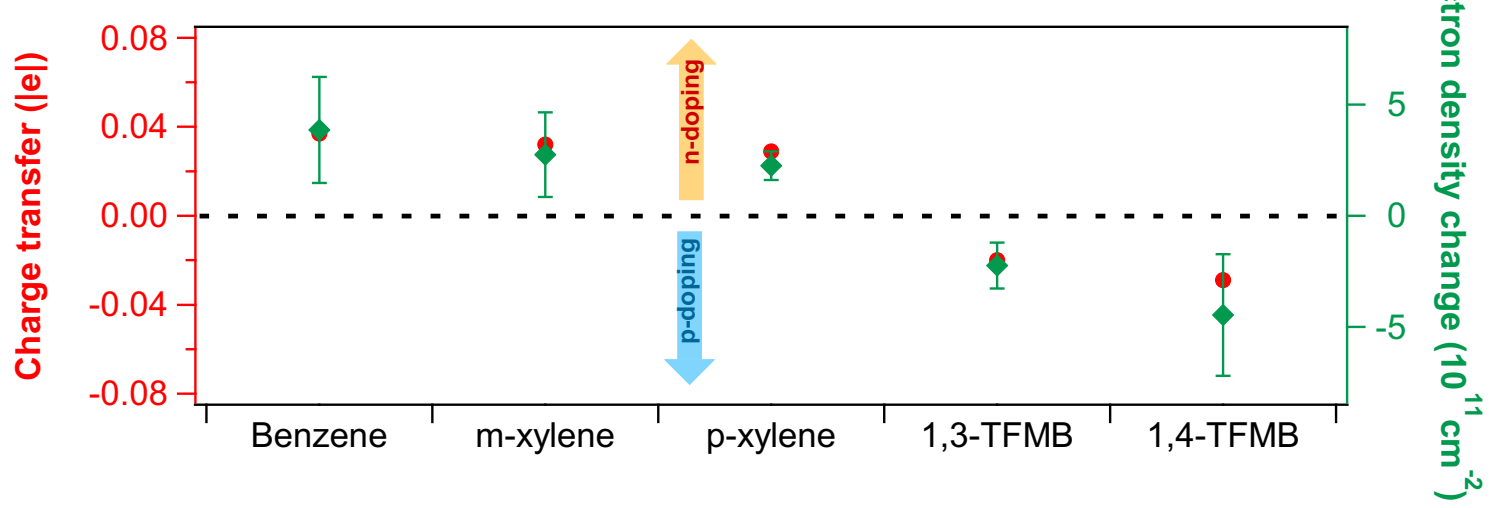

Figure 3. Electron density change (green) after physisorption on $\mathrm{MoS}_{2}$ of each aromatic molecule, as calculated by mass action model and provided by a DFT/Bader charge analysis (red).

\begin{tabular}{llllll}
\hline & Benzene & m-Xylene & p-Xylene & 1,3-TFMB & 1,4-TFMB \\
\hline Number of physisorbed molecules $\left(\mathbf{1 0}^{13} / \mathrm{cm}^{\mathbf{2}}\right)$ & 1.04 & 0.86 & 0.77 & 1.12 & 1.54 \\
\hline
\end{tabular}

Table 1 Estimated number of physisorbed aromatic molecules on monolayer $\mathrm{MoS}_{2}$ calculated from electron density change and transferred charge.

In contrast to monolayer $\mathrm{MoS}_{2}$, the photoluminescence behavior of monolayer $\mathrm{WSe}_{2}$ exhibits multiple possibilities for trion recombination. At low temperature, the coexistence of neutral excitons and positively charged excitons or negatively charged excitons and biexcitons is observed by changing temperature $(4 \mathrm{~K}, 10 \mathrm{~K}, 30 \mathrm{~K}, 60 \mathrm{~K})$ and device structure. ${ }^{3.33}$ To investigate the influence of aromatic molecules deposited on monolayer $\mathrm{WSe}_{2}$, we first study here its excitonic characteristics at $78 \mathrm{~K}_{\text {on }} \mathrm{SiO}_{2}$ substrate. The temperature dependence of monolayer $\mathrm{WSe}_{2}$ is similar to $\mathrm{MoS}_{2}$ whereby, 
upon decreasing the temperature, the exciton peak is blue-shifted. Defect-induced emissions appear below $200 \mathrm{~K}$ as multiple peaks in the range of $1.60 \mathrm{eV}$ to $1.65 \mathrm{eV}$ in which the intensity is dependent on the quality of the flakes. Moreover, at room temperature, a single excitonic peak at $1.66 \mathrm{eV}$ is observed. Below $100 \mathrm{~K}$, this peak is split into two independent components at $\sim 1.72 \mathrm{eV}$ and $\sim 1.70 \mathrm{eV}$ (Figure $\mathbf{S 2}$ in the Supporting Information).
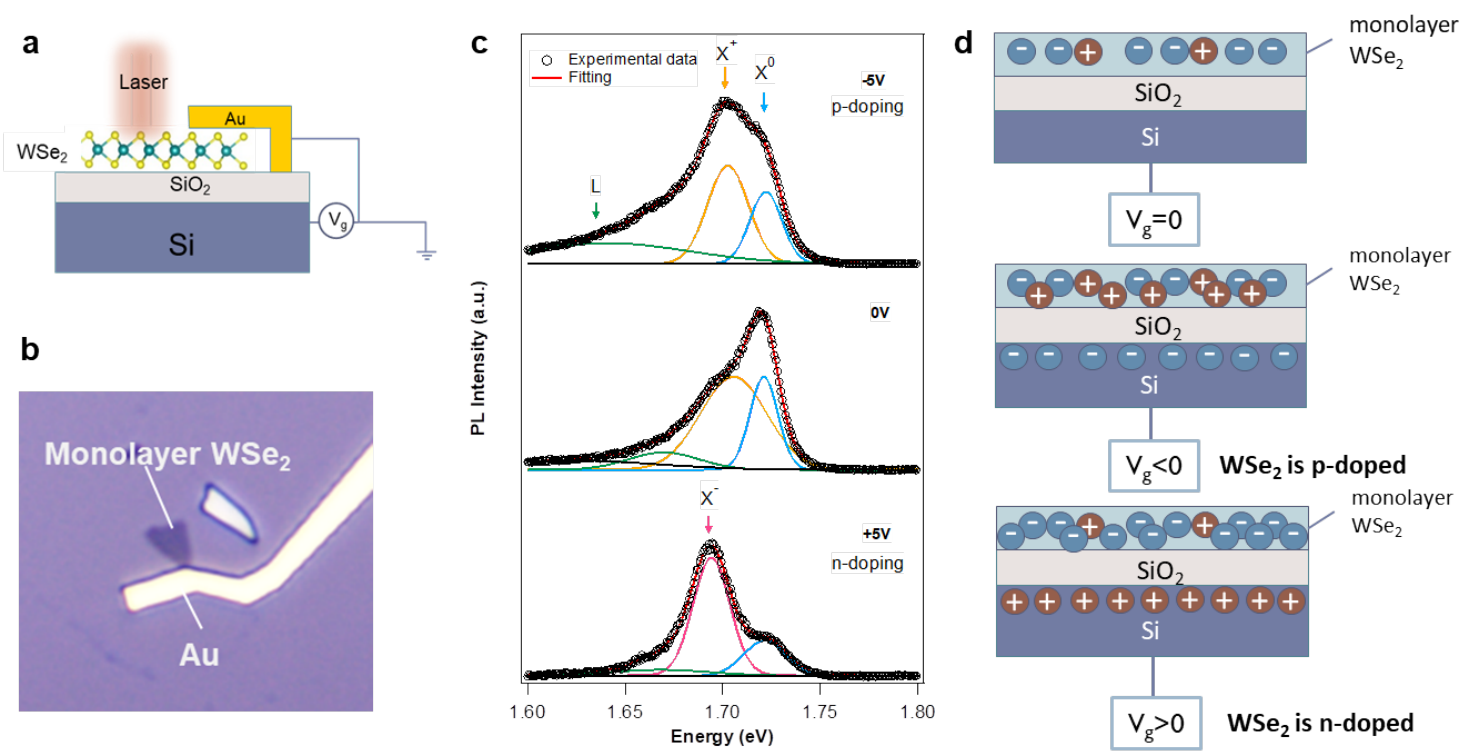

Figure 4. Gate-induced photoluminescence of monolayer $\mathrm{WSe}_{2}$. a) Schematic representation of the device for gate-induced photoluminescence in monolayer $\mathrm{WSe}_{2}$, and b) its corresponding optical image. c) PL spectra of monolayer $\mathrm{WSe}_{2}$ at $78 \mathrm{~K}$ under $5,0,+5 \mathrm{~V}$, demonstrating the recombination of neutral exciton $\left(\mathrm{X}^{\circ}\right)$ and positive trion $\left(\mathrm{X}^{+}\right)$at $0 \mathrm{~V}$ and $-5 \mathrm{~V}$, and the existence of neutral exciton $\left(\mathrm{X}^{\circ}\right)$ and negative trion $(\mathrm{X})$ at $+5 \mathrm{~V}$. d) Modulation of charge carrier by gating. The change in carrier density results in electrostatic doping on monolayer $\mathrm{WSe}_{2}$ and affects the recombination of positive or negative trions.

To assign the origin of these two excitonic components, field-effect devices were fabricated by E-beam lithography to induce electrical tunable doping. Figure 4(a) and (b) portray the schematic and optical image of the device, respectively. As displayed in the scheme in Figure 4(d), by adding a gate voltage on FET device, it is possible to modulate charge carrier density in $\mathrm{WSe}_{2}$ monolayers, and therefore to tune the 
proportion of excitons and trions. Upon applying negative gate voltage, electrons in monolayer $\mathrm{WSe}_{2}$ are attracted to the ground via the gold electrode, the electron density in the material is therefore largely decreased and neutral excitons tend to capture excess holes to form positive trion. On the contrary, when applying a positive gate voltage, more electrons are attracted to the channel, therefore negative trion recombination is facilitated. Figure 4(c) shows the PL spectra of monolayer $\mathrm{WSe}_{2}$ at $78 \mathrm{~K}$ under $-5 \mathrm{~V}, 0 \mathrm{~V}$ and $+5 \mathrm{~V}$ gate voltage, respectively. At $0 \mathrm{~V}$, apart from the peak at $\sim 1.65 \mathrm{eV}$, two other peaks, namely trion at $\sim 1.70 \mathrm{eV}$ and exciton at $\sim 1.72 \mathrm{eV}$ are observed. At $-5 \mathrm{~V}$, the intensity of the trion peak increases. Considering the increase in hole density by increasing negative gate voltage, we assign this peak as positive trion (two holes and one electron combined). This also implies that our material is intrinsically p-doped even after annealing, which is inconsistent with former works where either protection layer of $\mathrm{h}-\mathrm{BN}$ was added or the experiment was performed at lower temperature ${ }^{32}$. At $+5 \mathrm{~V}$, when more electrons are injected into monolayer $\mathrm{WSe}_{2}$, the negative trion (two electrons and one hole combined) emission at $\sim 1.69 \mathrm{eV}$ dominates. We extract the binding energy of positive trion and negative trion at $78 \mathrm{~K}$ to be 17 22 meV and 31 40 meV respectively by calculating the difference of trion emission energy and neutral exciton energy. We have also observed quantumconfined Stark effect by applying higher gate voltage and a substrate-induced hysteresis in PL mapping under small gating steps, yet this is beyond the scope of this work.

After studying the peak position of each type of exciton emission in our experimental conditions, we focus our attention on the effect of chemical doping on monolayer 
$\mathrm{WSe}_{2}$. With no gate voltage applied to the flake, we only discuss the neutral exciton and positive trion. We quantify the positive trion as

$$
\gamma^{+}=\frac{I_{X^{+}}}{I_{\text {total }}}=\frac{I_{X^{+}}}{I_{X}+I_{X^{+}}}
$$

where $\gamma^{+}$is the positive trion weight of monolayer $\mathrm{WSe}_{2}, I_{X^{+}}$is the area of positive trion peak, and $I_{X}$ is the area of neutral exciton peak and $I_{\text {total }}$ is the area of total photoluminescence intensity. Therefore, we calculate the trion weight change $\left(\Delta \gamma^{+}\right)$ to evaluate the charge transfer doping of molecules on monolayer $\mathrm{WSe}_{2}$.

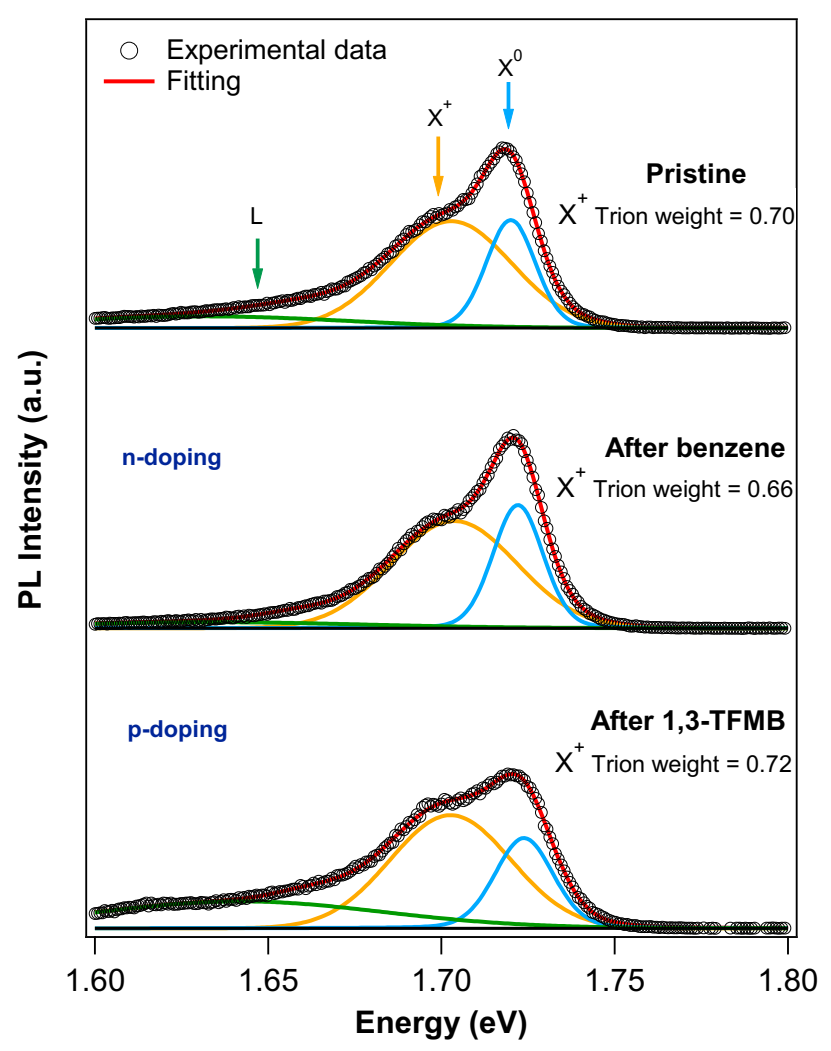

Figure 5. Photoluminescence spectra of monolayer $\mathrm{WSe}_{2}$ before and after physisorption of benzene, and 1,3-TFMB with calculated $\mathrm{X}^{+}$trion weight.

Figure 5 shows typical fitted PL spectra before and after doping with aromatic molecules at $78 \mathrm{~K}$. The spectral weight change of trion induced by benzene indicates n-doping and that 1,3-TFMB is a p-dopant. For other aromatic molecules, we find 
similar type of doping as monolayer $\mathrm{MoS}_{2}$ (detailed PL spectra in Figure S4 of Supporting Information). To elucidate the consistency of doping, we have calculated the chemical potential of monolayer $\mathrm{WSe}_{2}$ to be $-4.33 \mathrm{eV}$, which is nearly $1 \mathrm{eV}$ above the fluorinated molecules but still $1 \mathrm{eV}$ lower than the other molecules, suggesting that the charge transfer direction between the molecules and monolayer $\mathrm{WSe}_{2}$ should be the same as in $\mathrm{MoS}_{2}$. Both calculated charge transfers and trion weight changes reveal that benzene has the largest n-doping ability and that 1,4-TFMB p-dopes the monolayer $\mathrm{WSe}_{2}$ the most. Both xylenes have trivial influence on carrier density change in monolayer $\mathrm{WSe}_{2}$. (Figure 6)

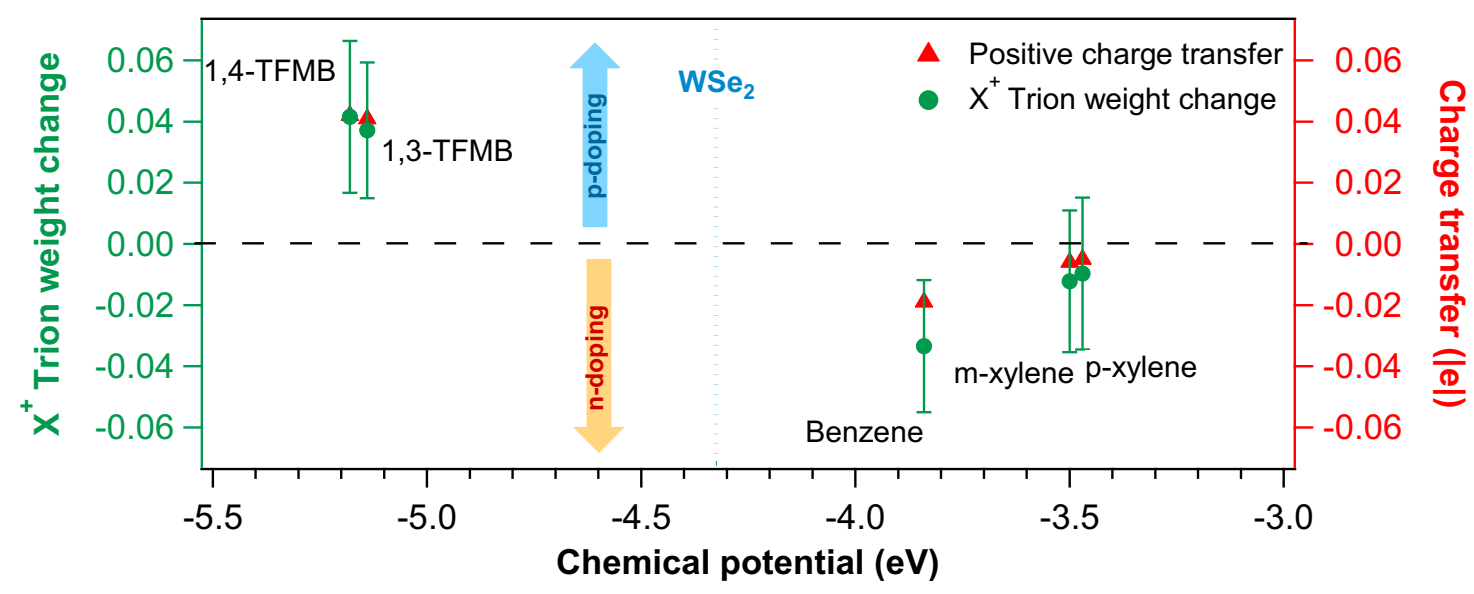

Figure 6. $\mathrm{X}^{+}$trion weight change and calculated DFT/Bader charge transfer (red) by physisorption of aromatic molecules from low-temperature PL measurements of monolayer $\mathrm{WSe}_{2}$ (green) as a function of chemical potential. Fluorinated molecules $(1,3-$ TFMB and 1,4-TFMB) possessing lower chemical potential are more prone to accept electrons from monolayer $\mathrm{MoS}_{2}$; in contrast, non-fluorinated molecules (benzene, $\mathrm{p}$ xylene and m-xylene) with higher chemical potential donate electrons to monolayer $\mathrm{WSe}_{2}$.

In summary, our spectroscopic investigation provides unambiguous evidence of the full potential of the physisorption of small aromatic molecules on $\mathrm{MoS}_{2}$ and $\mathrm{WSe}_{2}$ to tune their opto-electronic properties via charge transfer. The PL study performed at 78 $\mathrm{K}$ demonstrated for the first time that tunable chemical doping can be achieved on 
both 2D materials through a subtle choice of simple aromatic molecules and their dosing on the surface. In particular, while fluorinated aromatics determined a pdoping, an n-doping was observed for the other methyl-substituted molecules. The calculated charge transfer of fluorinated solvent molecules on $\mathrm{MoS}_{2}$ could be comparable to traditional organic p-dopants tetracyanoquinodimethane (TCNQ) and tetracyanoethylene (TCNE), while typical n-dopants tetrathiafuvaluene (TTF) and benzene viologen (BV) could donate electrons beyond the ability of aromatic solvents. ${ }^{17}$ Charge carrier modulation by optical response is found to be two orders of magnitude lower in charge carrier density than previous studies at room temperature in air $^{9}, 30$ by freezing the system at low temperature in inert atmosphere. The combined experimental analyses and DFT calculations utilized in this study represents a novel technique for estimating the density of physisorbed molecules in 2D surfaces. Furthermore, we have investigated for the first time the gate-tunable photoluminescence and defined binding energy of both positive and negative trions of monolayer $\mathrm{WSe}_{2}$ at $78 \mathrm{~K}$ through field-effect transistor.

Our results clearly indicate that care should be taken when choosing the solvent for the cleaning of the 2D materials or as "dispersant" for their chemical functionalization with larger (macro)molecules, since it can introduce strong electronic effects like doping. On the other hand, solvent molecules are clearly multifunctional systems since they both act as dispersant for the $2 \mathrm{D}$ materials and can enable the tuning of their optoelectronic properties.

Overall, our findings are instrumental both for fundamental and more applicative studies as many relevant solvents consist of small aromatic molecules, which therefore cannot be considered as inert media in the processing, but rather as a powerful tool for tuning the TMD properties and device optimization. 


\section{EXPERIMENTAL SECTION}

Sample preparation. Monolayer $\mathrm{MoS}_{2}$ and $\mathrm{WSe}_{2}$ flakes were mechanically exfoliated from commercially available molybdenum disulfide (Furuchi, Japan) and tungsten disulfide (HQ Graphene) crystals using the scotch tape method. The flakes were transferred onto $\mathrm{SiO}_{2}(90 \mathrm{~nm}) / \mathrm{Si}$ substrate, and their thickness was monitored by optical microscope combined with Raman spectroscopy and Atomic Force Microscopy (AFM). The samples were thermally annealed at $200{ }^{\circ} \mathrm{C}$ inside a vacuum chamber to desorb atmospheric adsorbates. Then, they were no longer exposed to air after the annealing and were characterized only under inert atmosphere $\left(\mathrm{N}_{2}\right.$-filled glovebox $)$. Anhydrous solvents from Sigma Aldrich were opened in glovebox filled with $\mathrm{N}_{2}$. To exclude the dielectric screening caused by environmental changes after depositing solvent molecules, we drop-cast each solvent molecule on monolayer TMDs, and spindried at $2000 \mathrm{RPM}$ for $60 \mathrm{~s}$ to guarantee the presence of limited number molecules physisorbed on the surface of the TMD.

Low-temperature photoluminescence spectroscopy. Photoluminescence spectra were recorded in inert atmosphere $\left(\mathrm{N}_{2}\right)$ by using a Renishaw inVia spectrometer equipped with $532 \mathrm{~nm}$ laser in aid of Linkam TP95 temperature controller. Samples were mounted in the glovebox and immediately measured after annealing to avoid exposure to air. The spectra were taken a different temperature, spanning from room temperature (298 K) down to $78 \mathrm{~K}$. The excitation power was kept below $1 \mathrm{~mW}$ to avoid local heating damage effects. The wavenumber (energy) resolution amounted to $\sim 1 \mathrm{meV}$.

Device fabrication and electrical characterization. Back-gated FETs were fabricated on thermally oxidized heavily $n$-doped silicon substrates (Fraunhofer Institute IPMS, $\rho_{\mathrm{Si}}$ $\left.\sim 0.001 \Omega \cdot \mathrm{cm}, t_{\mathrm{ox}}=90 \mathrm{~nm}\right)$ by means of E-beam lithography with polymethyl methacrylate (PMMA) resists, thermal evaporation of $\mathrm{Au}(80 \mathrm{~nm})$ and lift-off in acetone. Devices were annealed in high vacuum $\left(\sim 10^{-7}\right.$ mbar) overnight at $200{ }^{\circ} \mathrm{C}$ (Plassys 
ME400B). Electrical characterization was carried out at room temperature under $\mathrm{N}_{2}$ atmosphere (glovebox) with source-measurement units from Keithley (model 2636A).

Computational details. Our theoretical calculations were performed using density functional theory with the projector-augmented wave (PAW) scheme, as implemented in the Vienna Ab-Initio Simulation Package (VASP). ${ }^{34-35}$ The exchange-correlation potentials were treated by Perdew-Burke-Ernzerhof (PBE) ${ }^{36}$ parametrization of the generalized gradient approximation (GGA) and the kinetic energy cutoff for basis set was $600 \mathrm{eV}$. Van der Waals interactions were taken into account using Grimme's semiempirical DFT-D2 corrections. ${ }^{37}$ To model the physisorption of solvents on $\mathrm{MoS}_{2}\left(\mathrm{WSe}_{2}\right)$ monolayer, a $5 \times 5 \times 1$ supercell was constructed and a vacuum of $25 \AA$ thickness was used to avoid any physical interactions in the stacking direction. The first Brillouin zone integration was performed using $2 \times 2 \times 1$ and $4 \times 4 \times 1$ MonkhorstPack k-point mesh ${ }^{38}$ for geometry optimizations and electronic structure calculations, respectively. The integral atomic positions were fully relaxed according to the Hellmann-Feyman forces until the residual forces and total energy difference remain below $1 \mathrm{meV} / \AA$ and $10^{-5} \mathrm{eV}$, respectively.

\section{ASSOCIATED CONTENT}

The Supporting Information is available free of charge on the ACS Publications website at DOI:

\section{AUTHOR INFORMATION}

YW, SB and PS conceived the experiment. YW worked on sample preparation, device fabrication, optical and electrical characterization. YW, MAS and SB analyzed the data. AS, JC and DB performed the modeling work. YW and PS co-wrote the paper. All 
authors discussed the results and contributed to the interpretation of data as well as to editing the manuscript.

\section{ACKNOWLEDGEMENTS}

We thank Y. Zhao (ISIS) for valuable discussions and precious support. Device fabrication was carried out in part at the nanotechnology facility eFab (IPCMS, Strasbourg). The authors are thankful to S. Siegwald for assistance with microfabrication. We acknowledge funding from the M-ERA.NET project MODIGLIANI, the European Commission through the Graphene Flagship (GA696656) and the Marie-Curie IEF MULTI2DSWITCH (GA-700802), as well as the the Agence Nationale de la Recherche through the Labex projects CSC (ANR- 10LABX-0026 CSC) and NIE (ANR-11-LABX-0058 NIE) within the Investissement d'Avenir program (ANR-10-120 IDEX-0002-02), and the International Center for Frontier Research in Chemistry (icFRC). The work in Mons is supported by

FNRS/F.R.S. JC and DB are FNRS Research Directors.

\section{REFERENCES}

(1) Akinwande, D.; Petrone, N.; Hone, J. Two-dimensional flexible nanoelectronics. Nat. Commun. 2014, 5, 5678.

(2) Fiori, G.; Bonaccorso, F.; Iannaccone, G.; Palacios, T.; Neumaier, D.; Seabaugh, A.; Banerjee, S. K.; Colombo, L. Electronics Based on Two-Dimensional Materials. Nat. Nanotechnol. 2014, 9 (10), 768-779.

(3) Mak, K. F.; Shan, J. Photonics and optoelectronics of 2D semiconductor transition metal dichalcogenides. Nat. Photonics 2016, 10, 216.

(4) Hu, Y.; Huang, Y.; Tan, C.; Zhang, X.; Lu, Q.; Sindoro, M.; Huang, X.; Huang, W.; Wang, L.; Zhang, H. Two-dimensional transition metal dichalcogenide nanomaterials for biosensing applications. Mater. Chem. Frontiers 2017, 1 (1), 24-36.

(5) Li, S.-L.; Tsukagoshi, K.; Orgiu, E.; Samorì, P. Charge transport and mobility engineering in two-dimensional transition metal chalcogenide semiconductors. Chem. Soc. Rev. 2016, 45 (1), 118-151.

(6) Yan, W.; Txoperena, O.; Llopis, R.; Dery, H.; Hueso, L. E.; Casanova, F. A two-dimensional spin field-effect switch. Nat. Commun. 2016, 7, 13372. 
(7) Anichini, C.; Czepa, W.; Pakulski, D.; Aliprandi, A.; Ciesielski, A.; Samorì, P. Chemical sensing with 2D materials. Chem. Soc. Rev. 2018, 47, 4860-4908.

(8) Bertolazzi, S.; Gobbi, M.; Zhao, Y.; Backes, C.; Samorì, P. Molecular chemistry approaches for tuning the properties of two-dimensional transition metal dichalcogenides. Chem. Soc. Rev., 2018, 47,6845-6888.

(9) Mouri, S.; Miyauchi, Y.; Matsuda, K. Tunable photoluminescence of monolayer MoS2 via chemical doping. Nano Lett. 2013, 13 (12), 5944-5948.

(10) Lee, I.; Rathi, S.; Li, L.; Lim, D.; Khan, M. A.; Kannan, E. S.; Kim, G.-H. Nondegenerate n-type doping by hydrazine treatment in metal work function engineered WSe2 field-effect transistor. Nanotechnology 2015, 26 (45), 455203.

(11) Kiriya, D.; Tosun, M.; Zhao, P.; Kang, J. S.; Javey, A. Air-stable surface charge transfer doping of MoS2 by benzyl viologen. J. Am. Chem. Soc. 2014, 136 (22), 7853 7856.

(12) Splendiani, A.; Sun, L.; Zhang, Y. B.; Li, T. S.; Kim, J.; Chim, C. Y.; Galli, G.; Wang, F. Emerging Photoluminescence in Monolayer $\mathrm{MoS}_{2}$. Nano Lett. 2010, 10 (4), 1271-1275.

(13) Mak, K. F.; Lee, C.; Hone, J.; Shan, J.; Heinz, T. F. Atomically Thin $\mathrm{MoS}_{2}$ : A New Direct-Gap Semiconductor. Phys. Rev. Lett. 2010, 105 (13).

(14) Ross, J. S.; Wu, S.; Yu, H.; Ghimire, N. J.; Jones, A. M.; Aivazian, G.; Yan, J.; Mandrus, D. G.; Xiao, D.; Yao, W. Electrical control of neutral and charged excitons in a monolayer semiconductor. Nat. Commun. 2013, 4, 1474.

(15) Tongay, S.; Zhou, J.; Ataca, C.; Liu, J.; Kang, J. S.; Matthews, T. S.; You, L.; Li, J.; Grossman, J. C.; Wu, J. Broad-range modulation of light emission in twodimensional semiconductors by molecular physisorption gating. Nano Lett. 2013, 13 (6), 2831-6.

(16) Gobbi, M.; Orgiu, E.; Samorì, P. "When 2D Materials Meet Molecules: Opportunities and Challenges of Hybrid Organic/Inorganic van der Waals Heterostructures. Adv. Mater. 2018, 30, 1706103.

(17) Jing, Y.; Tan, X.; Zhou, Z.; Shen, P. W. Tuning electronic and optical properties of MoS2 monolayer via molecular charge transfer. J. Mater. Chem. A 2014, 2 (40), 16892-16897.

(18) Han, H. V.; Lu, A. Y.; Lu, L. S.; Huang, J. K.; Li, H.; Hsu, C. L.; Lin, Y. C.; Chiu, M. H.; Suenaga, K.; Chu, C. W.; Kuo, H. C.; Chang, W. H.; Li, L. J.; Shi, Y. Photoluminescence enhancement and structure repairing of monolayer $\mathrm{MoSe} 2$ by hydrohalic acid treatment. ACS Nano 2016, 10 (1), 1454-1461.

(19) de la Rosa, C. J. L.; Phillipson, R.; Teyssandier, J.; Adisoejoso, J.; Balaji, Y.; Huyghebaert, C.; Radu, I.; Heyns, M.; De Feyter, S.; De Gendt, S. Molecular doping of MoS2 transistors by self-assembled oleylamine networks. Appl. Phys. Lett. 2016, $109(25), 253112$.

(20) Kang, D. H.; Dugasani, S. R.; Park, H. Y.; Shim, J.; Gnapareddy, B.; Jeon, J.; Lee, S.; Roh, Y.; Park, S. H.; Park, J. H. Ultra-low Doping on Two-Dimensional Transition Metal Dichalcogenides using DNA Nanostructure Doped by a Combination of Lanthanide and Metal Ions. Sci. Rep. 2016, 6, 20333. 
(21) Fang, H.; Chuang, S.; Chang, T. C.; Takei, K.; Takahashi, T.; Javey, A. Highperformance single layered WSe2 p-FETs with chemically doped contacts. Nano Lett. 2012, 12 (7), 3788-3792.

(22) Fisher, A.; Blöchl, P. Adsorption and scanning-tunneling-microscope imaging of benzene on graphite and MoS 2. Phys. Rev. Lett. 1993, 70 (21), 3263.

(23) Dong, X.; Fu, D.; Fang, W.; Shi, Y.; Chen, P.; Li, L. J. Doping single - layer graphene with aromatic molecules. Small 2009, 5 (12), 1422-1426.

(24) Korn, T.; Heydrich, S.; Hirmer, M.; Schmutzler, J.; Schüller, C. Lowtemperature photocarrier dynamics in monolayer MoS2. Appl. Phys. Lett. 2011, 99 (10), 102109.

(25) Lanzillo, N. A.; Birdwell, A. G.; Amani, M.; Crowne, F. J.; Shah, P. B.; Najmaei, S.; Liu, Z.; Ajayan, P. M.; Lou, J.; Dubey, M.; Nayak, S. K.; O'Regan, T. P. Temperature-dependent phonon shifts in monolayer MoS2. Appl. Phys. Lett. 2013, $103(9), 093102$.

(26) Mak, K. F.; He, K. L.; Lee, C.; Lee, G. H.; Hone, J.; Heinz, T. F.; Shan, J. Tightly bound trions in monolayer MoS2. Nat. Mater. 2013, 12 (3), 207-211.

(27) Crispin, X.; Geskin, V.; Crispin, A.; Cornil, J.; Lazzaroni, R.; Salaneck, W. R.; Bredas, J.-L. Characterization of the interface dipole at organic/metal interfaces. $J$. Am. Chem. Soc. 2002, 124 (27), 8131-8141.

(28) Pearson, R. G. The electronic chemical potential and chemical hardness. J. Mol. Struct.: THEOCHEM 1992, 255, 261-270.

(29) Tang, W.; Sanville, E.; Henkelman, G. A grid-based Bader analysis algorithm without lattice bias. J. Phys. Cond. Matter. 2009, 21 (8), 084204.

(30) Choi, J.; Zhang, H.; Du, H.; Choi, J. H. Understanding solvent effects on the properties of two-dimensional transition metal dichalcogenides. ACS Appl. Mater. Interfaces 2016, 8 (14), 8864-8869.

(31) Ross, J. S.; Klement, P.; Jones, A. M.; Ghimire, N. J.; Yan, J.; Mandrus, D.; Taniguchi, T.; Watanabe, K.; Kitamura, K.; Yao, W. Electrically tunable excitonic light-emitting diodes based on monolayer WSe 2 p-n junctions. Nat. Nanotechnol. 2014, 9 (4), 268.

(32) Jones, A. M.; Yu, H.; Ghimire, N. J.; Wu, S.; Aivazian, G.; Ross, J. S.; Zhao, B.; Yan, J.; Mandrus, D. G.; Xiao, D. Optical generation of excitonic valley coherence in monolayer WSe 2. Nat. Nanotechnol. 2013, 8 (9), 634.

(33) You, Y.; Zhang, X.-X.; Berkelbach, T. C.; Hybertsen, M. S.; Reichman, D. R.; Heinz, T. F. Observation of biexcitons in monolayer WSe 2. Nat. Phys. 2015, 11 (6), 477.

(34) Kresse, G.; Furthmüller, J. Efficient iterative schemes for ab initio total-energy calculations using a plane-wave basis set. Phys. Rev. B 1996, 54 (16), 11169.

(35) Kresse, G. G. Kresse and D. Joubert, Phys. Rev. B 59, 1758 (1999). Phys. Rev. $B$ 1999, 59, 1758.

(36) Perdew, J. P.; Burke, K.; Ernzerhof, M. Generalized gradient approximation made simple. Phys Rev Lett 1996, 77 (18), 3865. 
(37) Grimme, S. Semiempirical GGA - type density functional constructed with a long - range dispersion correction. J. Comput. Chem. 2006, 27 (15), 1787-1799.

(38) Monkhorst, H. J.; Pack, J. D. Special points for Brillouin-zone integrations. Phys. Rev. B 1976, 13 (12), 5188. 